\section{INVESTIGATION OF DEATHS FOLLOWING COVID-19 VACCINATION IN WESTERN AUSTRALIA}

\author{
Kirralee Patton, Leana Downs, Clive Cooke, Jodi White, \\ Victoria Kueppers \\ Forensic Pathology, PathWest Laboratory Medicine, QEII, \\ Perth, WA, Australia
}

Coronavirus disease (COVID-19) is an infectious disease caused by the severe acute respiratory syndrome corona virus 2 (SARSCoV-2). The World Health Organization declared a COVID-19 pandemic in March 2020 and mass vaccination programmes started in early December 2020.

Vaccination against COVID-19 is the most effective way to reduce deaths and severe illness from infection, and the protective benefits of vaccination continue to outweigh the potential risks. However, like all medicines, COVID-19 vaccines may cause some side effects, most of which are mild such as a sore arm at the injection-site, headache, muscle pain, fever and chills. Unfortunately, a few very rare but serious side effects have been linked to COVID-19 vaccinations: thrombosis with thrombocytopenia syndrome, Guillain-Barré syndrome, immune thrombocytopenia and capillary leak syndrome following AstraZeneca, and myocarditis and pericarditis following Pfizer and Moderna. ${ }^{1}$ As of 12 December 2021, approximately 40.2 million total COVID-19 vaccine doses have been administered, across Australia. ${ }^{1}$ The TGA has identified 11 reports of death that were linked to AstraZeneca vaccination: eight cases of thrombosis with thrombocytopenia syndrome, two cases linked to GuillainBarré syndrome and one case of immune thrombocytopenia. ${ }^{1}$ We would like to present the Western Australian experience of investigating cause of death following COVID-19 vaccination, including clinical criteria and laboratory testing.

\section{Reference}

1. Australian Government Department of Health: Therapeutic Goods Administration. COVID-19 vaccine weekly safety report - 16-12-2021. Cited 16 Dec 2021. https://www.tga.gov.au/ periodic/covid-19-vaccine-weekly-safety-report-16-12-2021.

\section{MANAGEMENT OF DEATHS IN TEMPORAL RELATIONSHIP TO COVID-19 IMMUNISATION IN NSW}

Isabel Brouwer, Dianna Inmon, Angela Duncan

Forensic Medicine Service, Forensic and Analytical Science Service, NSW Health Pathology, NSW, Australia

With the emergence of the COVID-19 pandemic and subsequent widespread disruption, the world waited in anticipation for the development of vaccines. The rapid development of vaccines and the introduction of immunisation programs in NSW raised public safety concerns and initiated intense media scrutiny. The NSW State Coroner declared all deaths suspected to be due to an adverse event following immunisation are reportable under the NSW Coroners Act. Very little was known at the time about the adverse events that may follow immunisation and how these deaths may present to the Coroner. A temporally associated death was defined as occurring within 6 weeks of vaccination where it is plausible that vaccination contributed to or caused the conditions resulting in death.

Forensic Medicine service played an essential role in the broader NSW Health's COVID-19 vaccine safety surveillance strategy.
This presentation will focus on the development of clinical pathways within the NSW Coronial process to support and enhance clinical decision making and how these pathways evolved with our emergent knowledge of adverse events following COVID immunisation. Reference will also be made to interdependencies with stakeholders and the unique challenges of these collaborations

\section{THE ROLE OF THE MATERNAL DEATH REVIEW}

\section{Matthew J. Lynch, Joanna M. Glengarry}

Department of Forensic Medicine, Monash University, Melbourne, Vic, Australia, Victorian Institute of Forensic Medicine Southbank, Vic, Australia

Maternal mortality review committees are statutory authorities involved in data collection and research. They undertake reviews of all cases of maternal mortality and increasingly examine instances of significant maternal morbidity. Their bailiwick includes all maternal deaths occurring during pregnancy and within a year of birth. Cases of significant morbidity are identified based on intensive care admissions during pregnancy and the 42 days of the puerperium. The committees classify deaths as direct (relating to the pregnancy or birth, e.g., amniotic fluid embolism), indirect (relating to pre-existing medical conditions, e.g., valvular heart disease), incidental (unrelated to the pregnancy or birth, e.g., motor vehicle collisions) or late (deaths occurring between 42 days and 1 year). The aim of the review process is to identify trends in maternal mortality and morbidity and to identify opportunities for intervention. To this end the committees produce annual reports containing recommendations and so-called 'good practice points'. Committees invariably comprise practitioners from a range of specialities including obstetrics, mid-wifery, intensive care, anaesthetics, psychiatry, and pathology (obstetric/perinatal/forensic). Forensic pathologists play a crucial role in these reviews by identifying deaths which might otherwise go undetected, demystifying the jargon of the necropsy report and assisting the committee to navigate the labyrinthine Coronial death investigation process.

\section{References}

1. Australian Institute of Health and Welfare (AIHW). Maternal Deaths in Australia 2015-2017. Cat. no. PER 106. Canberra: AIHW, 2020.

2. Australian Institute of Health and Welfare (AIHW). Maternal deaths in Australia. Cat. no. PER 99. Cited 10 Dec 2021. http://www.aihw.gov.au/reports/mothers-babies/maternaldeaths-in-australia

3. Consultative Council on Obstetric and Paediatric Mortality and Morbidity. Victoria's Mothers, Babies, and Children 2019. Melbourne: Victorian Government, Safer Care Victoria, $2021 ; 20-3$.

\section{NEW AND NOVEL TOXICOLOGICAL AGENTS}

Peter Stockham $^{1,2}$

${ }^{1}$ Forensic Science SA, Adelaide, SA, Australia; ${ }^{2}$ Flinders University, Bedford Park, SA, Australia

New psychoactive substances (NPS) are synthetic toxicological agents that mimic the effects of existing drugs of abuse (DOA), 